\title{
Evaluación de la presencia de hongos micorrízico arbusculares en un bosque de pino-encino en Chiapas, México
}

\author{
Evaluation of mycorrhizal fungi in a pine-oak forest in Chiapas, Mexico
}

\author{
Yolanda del Carmen Pérez-Luna1, Peggy Elizabeth Álvarez-Gutiérrez³, Daniel González Mendoza4, \\ Vianey Mendez-Trujillo ${ }^{2,4 *}$
}

\begin{abstract}
RESUMEN
Los hongos endomicorícicos son organismos importantes en la rizosfera del suelo. Constituyen productos no maderables cuyo uso sustentable deben tener una base ecológica. En este trabajo se evaluó el efecto que tiene la pinarización y la abundancia de hojarasca sobre el porcentaje de infección micorrízica en raíces de pino-encino ubicado en un bosque del cerro Ecatepec. Los porcentajes de infección fueron, en promedio, de $17.7 \%$ debido a que el trabajo se desarrolló en una temporada fresca y seca (febrero) encontrándose estructuras como hifas y vesículas. No se observó ningún efecto de la pinarización sobre el porcentaje de infección micorrízica, sin embargo la profundidad de hojarasca presentó un efecto estadísticamente significativo sobre la variable de infección, lo cual puede deberse a que la presencia de hojarasca beneficia la actividad microbiológica del suelo y además la humedad y temperatura de la capa de hojarasca favorece el desarrollo de hongos micorrízicos.
\end{abstract}

Palabras clave: pinarización, micorrizas, Quercus

\begin{abstract}
Micorrizical fungi are an importan organisms in forest rhizospere. They are non timber forest products whose sustainable use must have an ecological basis. Pinarization effect and abundance of litter was determined on the percentage of mycorrhizal infection in roots in a pine-oak forest located in Ecatepec hill. The infection rates were 17.7\% due to the work developed in a cool, dry season (February). The structures found in pine roots was as hyphae and vesicles. No effect of pinarización on the percentage of mycorrhizal infection was observed, however the depth of litter showed a statistically significant effect on the variable of infection, which may be due to the presence of litter benefits the soil microbial activity and also humidity and temperature of the litter layer favors the development of mycorrhizal fungi.
\end{abstract}

Keywords: pinarization, mycorrhizae, Quercus

\section{Introducción}

Los patrones de uso de suelo en los Altos de Chiapas han propiciado en décadas recientes la expansión de poblaciones de Pinus spp. en áreas que mantenían bosques de encino y mesófilo de montaña (González Espinosa, 2000). La pinarización entendida como el proceso de formación de bosques de pinos debidoala capacidad invasiva de estaespecie trae serias consecuencias a la diversidad. El aumento sucesivo en el dominio de pino de bosques es un proceso notable en Chiapas y en otras montañas tropicales de México. Los bosques ricos florísticamente con un dosel dominado por robles o de otros árboles de hoja caduca

1 Universidad Politécnica de Chiapas. Campus Suchiapa, México.

2 Tecnológico Nacional de México. Instituto Tecnológico Superior de Cintalapa. Chiapas, México.

3 Tecnológico Nacional de México. Campus Tuxtla Gutiérrez. Chiapas, México.

4 Instituto de Ciencias Agrícolas, Universidad Autónoma de Baja California (ICA-UABC). Ejido Nuevo León, Baja California, México.

* Autor para correspondencia: ibqvianey@yahoo.com; vianey.mendez@uabc.edu.mx

Fecha de recepción: 5 enero, 2019.

Fecha de aceptación: 22 febrero, 2019.

DOI: http://dx.doi.org/10.4067/S0718-34292019005000401. Publicado en línea: 13-junio-2019. 
se están convirtiendo en comunidades mucho menos diversas dominadas por pino. Los pinares inducidos varían más en su temperatura y humedad interiores y sus suelos son más compactos, y menos fértiles que los bosques contiguos de los cuales se derivan (GalindoJaimes et al., 2002). La importancia de los bosques de pino-encino se debe a que los pinos y encinos son los árboles más representativos y económicamente importantes de los ecosistemas templados de México y del mundo; de hecho, son la base misma de la mayor parte de la industria forestal del país. En bosques de pinos (Pinus spp.) y encinos (Quercus spp.) el suelo constituye un elemento fundamental, dado que cumple con importantes funciones, de las cuales se derivan servicios ambientales indispensables para el sostenimiento tanto del ecosistema como de la vida humana. La función más conocida es la de soporte y suministro de nutrientes a las plantas. Un componente importante dentro del reciclaje de nutrimentos lo constituyen las micorrizas. En ecosistemas naturales, las raíces de la mayoría de plantas están asociadas simbióticamente a hongos del suelo del orden Glomales (Morton 1990, Morton y Benny 1990) formando micorrizas del tipo arbuscular.

El mayor significado agronómico de estas micorrizas es el aumentar el volumen de suelo explorado por las raíces para tomar elementos de lenta difusión tales como fósforo, zinc y cobre, principalmente. Debido a que los suelos de los Altos de Chiapas evidencian una alta susceptibilidad a la erosión y una baja fertilidad natural (Álvarez-Solís y León Martínez, 1997) requieren la aplicación de fertilizantes químicos que compensen las deficiencias nutrimentales para asegurar la producción de cultivos (Álvarez-Solís, et al., 1998).

Debido a la creciente pinarización en Chiapas con este estudio se pretende evaluar el efecto de este proceso en la presencia de hongos formadores de micorrizas en las especies de pinos y encinos. Dadas las condiciones nutricionales del suelo en este sitio y los requerimientos para el establecimiento de las micorrizas es problable que existan diferencias en el porcentaje de micorrización, de manera que a medida que la pinarización aumenta se estimula la infección micorrízica en las raíces evaluadas principalmente por la acidificación del suelo ya que el hongo se desarrolla mejor a valores bajos de $\mathrm{pH}$ (3.5 - 4.2). Por lo tanto, el objetivo de este trabajo fue determinar el efecto de la abundancia de hojarasca y del índice de pinarización sobre la infección micotrófica en un bosque de pino-encino mediante la identificación de estructuras propias de los hongos micorrízicos.

\section{Metodología}

\section{Sitio de estudio}

El estudio se realizó en un bosque de pinoencino ubicado en el Cerro Ecatepec localizado en las afueras de la ciudad de San Cristóbal de Las Casas, Chiapas, México a los 16,719 y 92,663 grados de latitud y longitud, respectivamente (Figura 1). El suelo en este sitio corresponde a una Rendzina somera, con un horizonte mineral de $8-10 \mathrm{~cm}$ de profundidad sobre una cama altamente heterogénea de rocas. Los sitios seleccionados corresponden a un bosque de encino con disturbio moderado con árboles de 60 años (sitio 1), una zona con dominancia de pino con árboles de 50 años pero en su mayoría de 25 a 30 años (sitio 2) y un ecotono con presencia de ambas especies (sitio 3). El establecimiento de pino se debió a la limpieza que se realizó hace 50 años de una parte del bosque de encino para realizar agricultura de tala y quema, 3 años más tarde el campo fue abandonado para permitir la regeneración natural del mismo. La extracción selectiva de encinos a edades tempranas impiden la regeneración de los encinos y promueven la dominancia de pinos (García-Barrios, 2003).

\section{Diseño experimental}

Se realizó un diseño de tres tratamientos con bloques completamente al azar con 20 repeticiones en cada uno de los tres sitios que conforman el bosque de pino-encino, para tener un total de 60 unidades experimentales a lo largo de la ladera de dicho bosque.

\section{Toma de muestra}

La toma de muestra para su análisis se realizó en los primeros 8-10 $\mathrm{cm}$ de suelo superficial a 50 $\mathrm{cm}$ de distancia de tronco del árbol (pino o encino) utilizando un cilindro de acero inoxidable de $5 \mathrm{~cm}$ de diámetro, de manera que se tuvieran raíces para determinar la infección micorrízica en cada uno de los sitios previamente seleccionados. Se registraron datos de profundidad de hojarasca en cada unidad experimental considerándose el promedio de cinco mediciones, abundancia de hojarasca en un cuadrante

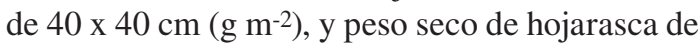
pino y hojarasca total para calcular el índice de 


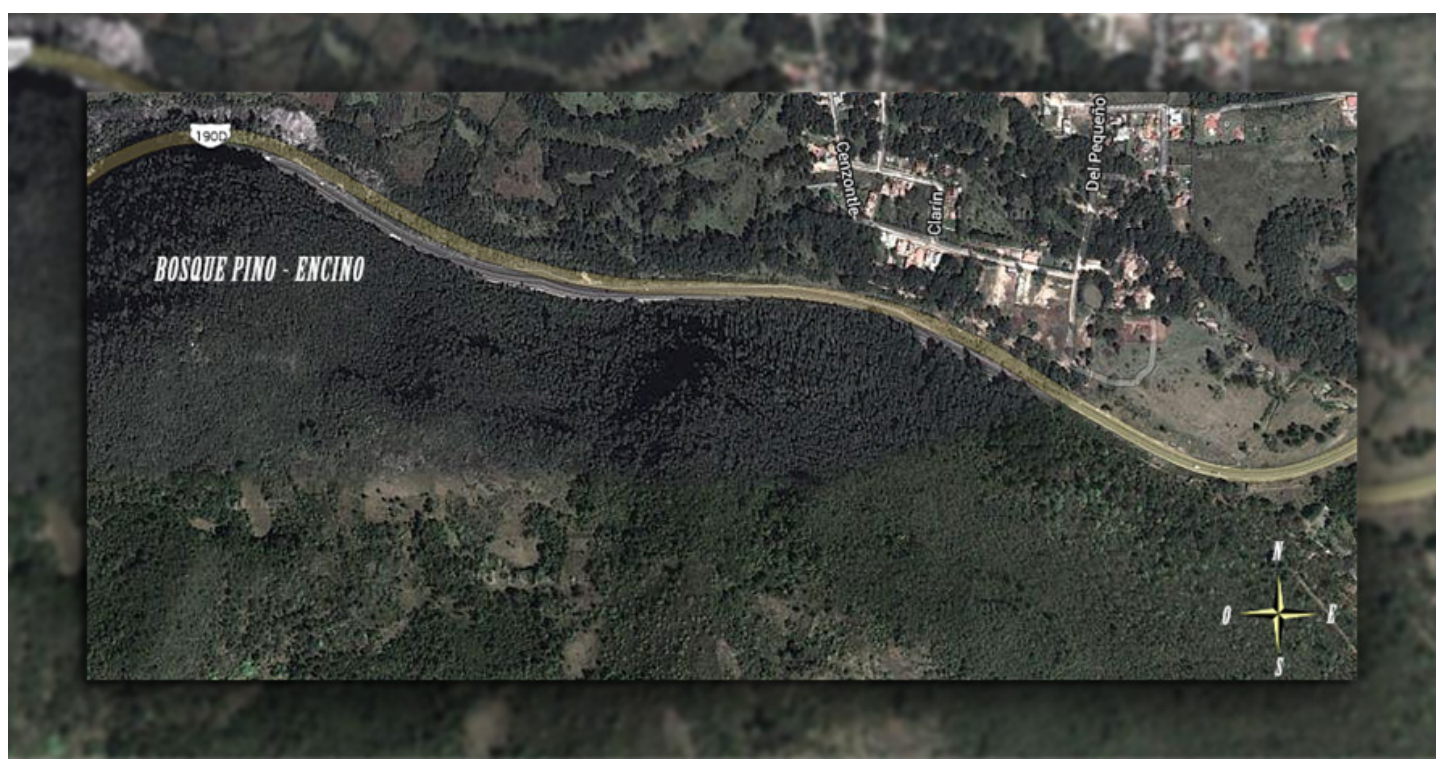

Figura 1. Bosque de pino - encino ubicado en el cerro Ecatepec, municipio de San Cristóbal de las Casas, Chiapas, México.

pinarización. Una vez que se extrajeron las muestras de suelo, fueron colocadas en bolsas para la posterior separación en laboratorio de las raicillas, mismas que fueron lavadas para eliminar restos de suelo y colocadas en frascos de plástico con solución de formaldehído (6,0\%), ácido acético (2,3\%), alcohol $(47,8 \%)$ y agua destilada $(43,9 \%)$ (FAAA) para su posterior procesamiento.

\section{Tinción de raíces y determinación del porcentaje de colonización}

Una vez preparadas las raíces se efectuó el procedimiento de clareo y tinción para observación de estructuras infectivas según el método de Phillips y Hayman (1979). La tinción de raíces se realiza a través de los siguientes pasos: clareo, blanqueo, acidificación, tinción y decoloración. El porcentaje de colonización se midió a partir de 10 fragmentos de raíz teñida de aproximadamente $1 \mathrm{~cm}$ de longitud por cada subparcela tomada, seleccionados al azar, registrando la presencia de estructuras propias del hongo por cada campo óptico de observación microscópica.

\section{Análisis estadístico}

Los datos fueron analizados utilizando el paquete estadístico JMP ® v. 7,0,1 (Copyright 2007 SAS Institute Inc.) y el programa SPSS v. 10, realizándose un análisis de correlación y análisis de regresión lineal para determinar el efecto de la abundancia de hojarasca y de la pinarización sobre el porcentaje de infección micorrízica, así como el efecto de la profundidad de la hojarasca.

\section{Resultados}

El análisis de las raíces reveló la presencia de hongos endomicorrízicos en el bosque de pinoencino evaluado, de manera que en encino (1) se presentó un $18,5 \%$ de infección, para pino (2) $17 \%$ y para el ecotono (3) 16\% de infección (Tabla 1). Además se identificaron estructuras de hongos micorrízicos principalmente hifas (Figura 2), cuya función es la toma y transferencia de nutrientes del sustrato hacia la planta; en pocos casos vesículas (Figura 3) y no se observaron arbúsculos. De manera general el porcentaje de infección micorrízica en el bosque de pino-encino fue de $17,17 \%$, con un IP medio de 54,77 y $887,94 \mathrm{~g} \mathrm{~m}^{-2}$ de abundancia de hojarasca (valor medio).

\section{Análisis de correlación}

De manera individual se procedió a analizar el porcentaje de infección micorrízica para las 60 unidades experimentales en función de la abundancia de hojarasca $\left(\mathrm{g} \mathrm{m}^{-2}\right)$ e índice de pinarización, 
Tabla 1. Porcentaje de micorrizacion e índice de pinarización en un bosque pino-encino

\begin{tabular}{lcc}
\hline Sitios de muestreo & Micorrizacion $(\%)$ & Índice de pinarización $\left(\mathrm{g} \mathrm{m}^{-2}\right)$ \\
\hline Sitio 1 (encino) & $18,5 \pm 0,27^{\mathrm{a}}$ & $887,94 \pm 16,54^{\mathrm{a}}$ \\
Sitio 2 (pino) & $17,0 \pm 0,75^{\mathrm{b}}$ & $54,94 \pm 8,10^{\mathrm{b}}$ \\
Sitio 3 (ecotono) & $16 \pm 0,45^{\mathrm{c}}$ & $295 \pm 9,24^{\mathrm{a}}$ \\
\hline Resulados son expresados como
\end{tabular}
( $\mathrm{a}, \mathrm{b}, \mathrm{o}$ c) en cada columna son iguales de acuerdo a Tukey test at $\mathrm{P} \leq 0,5$.
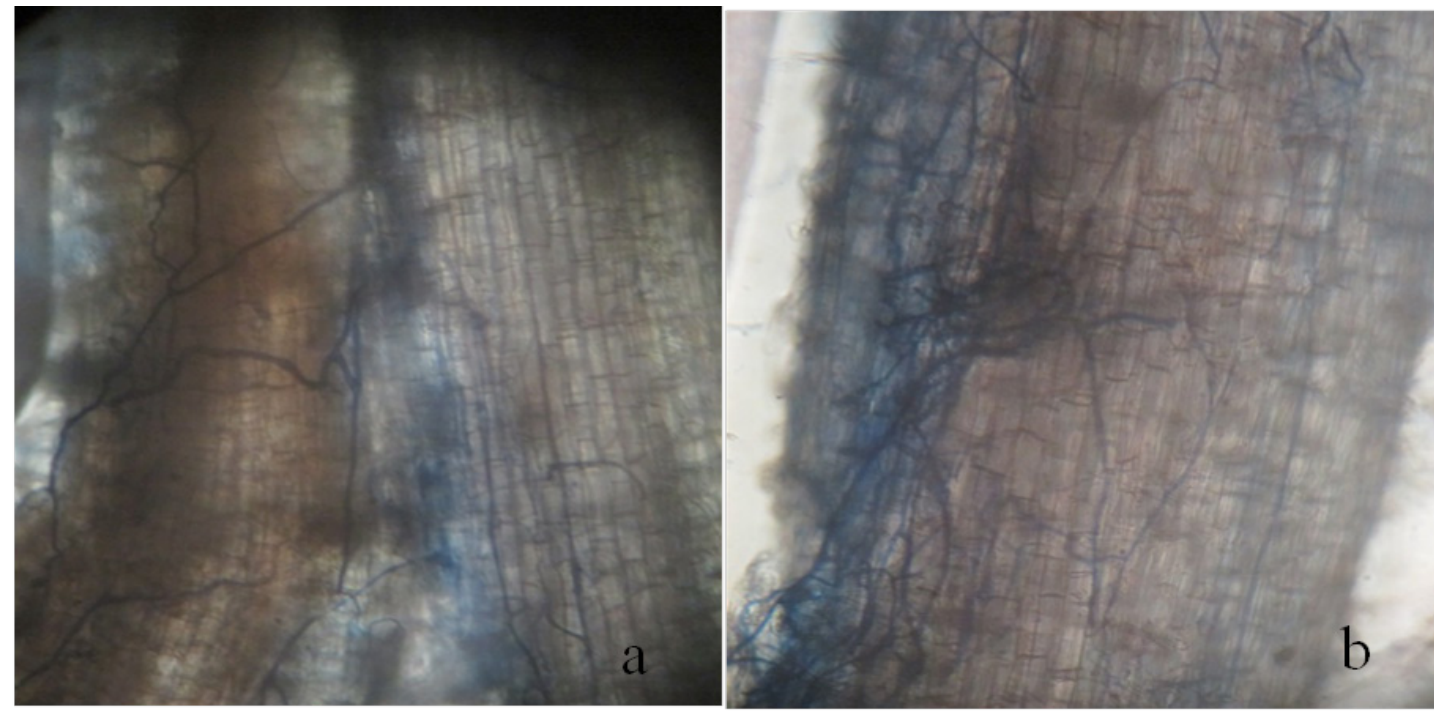

Figura 2. Presencia de hifas de hongos micorrízico arbusculares en raíces de a) pino y b) encino

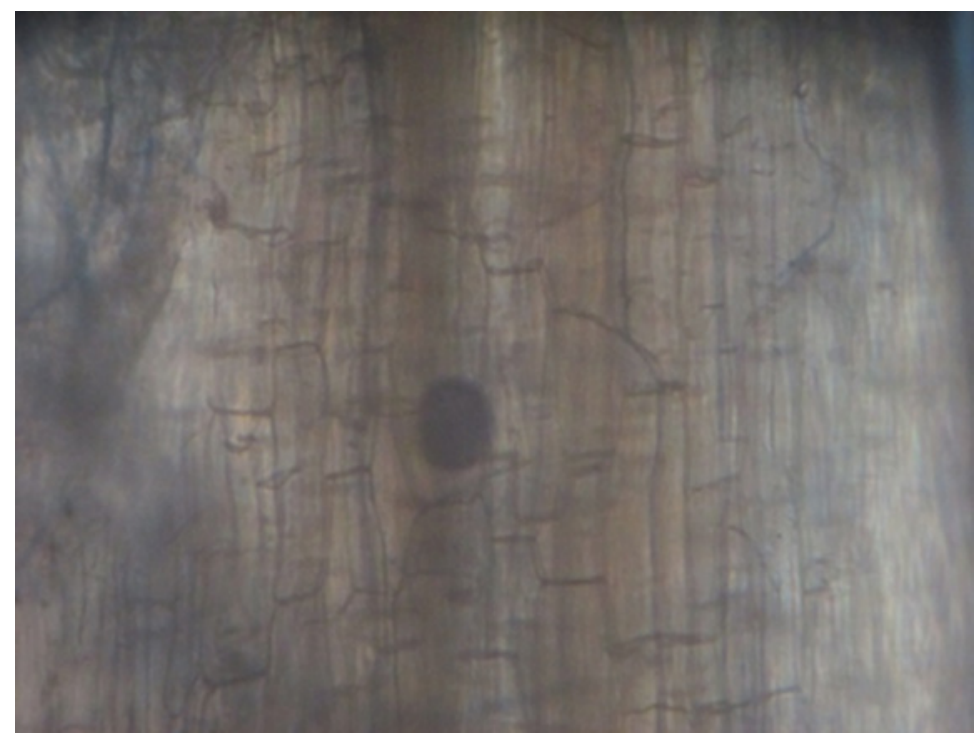

Figura 3. Vesículas de hongos micorrízico arbusculares en raíces de pino 


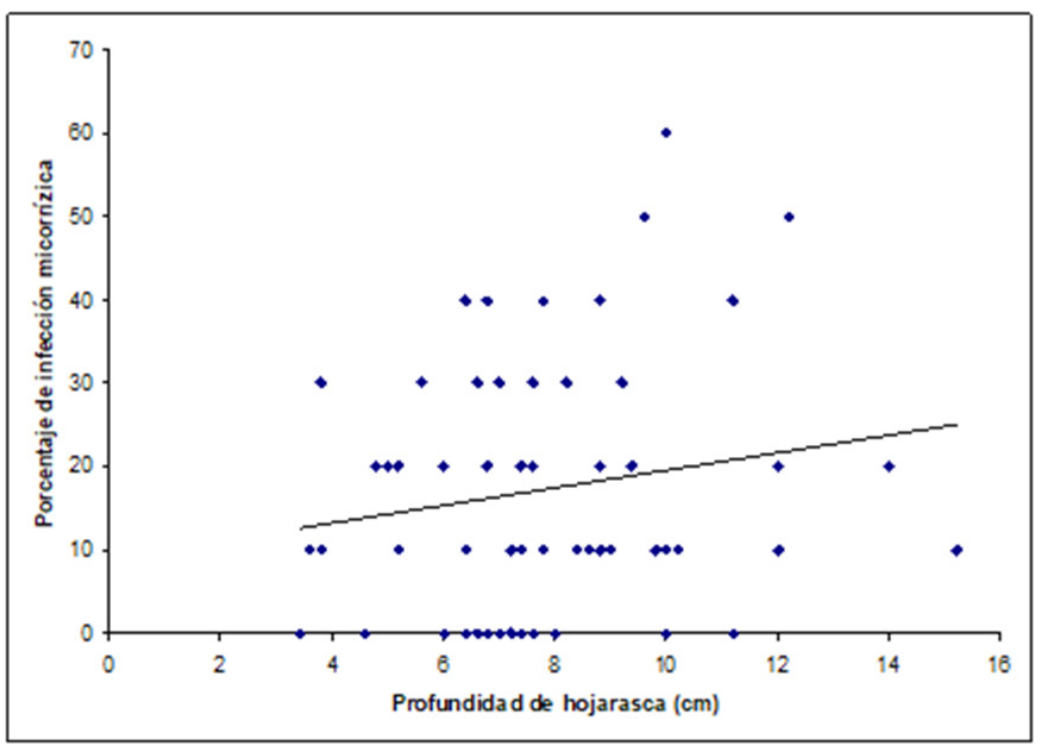

Figura 4. Análisis de correlación entre el porcentaje de infección y la profundidad de la hojarasca (cm).

realizándose un análisis de correlación. Tanto para infección micorrízica y abundancia de hojarasca como para infección micorrízica e índice de pinarización no existe correlación alguna $\left(\mathrm{R}^{2}=\right.$ 0,012361 y $R^{2}=0,001181$, respectivamente). Para el caso del porcentaje de infección contra la abundancia de hojarasca se observa que en el rango de $200 \mathrm{~g}$ $\mathrm{m}^{-2}$ a $1200 \mathrm{~g} \mathrm{~m}^{-2}$ de hojarasca, aproximadamente, se encuentran contenidas el mayor número de unidades experimentales presentando porcentajes de infección desde 20 a $60 \%$; por el contrario en cuanto a porcentaje de infección e índice de pinarización (IP) el grueso de los datos se ubican a valores más altos de IP observándose el porcentaje más alto de infección (60\%) en una sola unidad experimental. El análisis de correlación entre el porcentaje de infección micorrízica y la profundidad de la hojarasca reveló que aun cuando pareciera haber una tendencia de incremento en el porcentaje de infección conforme aumenta la profundidad de la hojarasca se obtuvo un valor de $\mathrm{r}^{2}=0,032$, lo cual indica que no existe correlación entre ambas variables (Figura 4).

\section{Análisis de Regresión lineal}

Con el análisis de regresión lineal se puede observar que el IP no tiene un efecto significativo sobre el porcentaje de infección micorrízica, contrario a lo observado para la profundidad de la hojarasca que tiene un efecto positivo y estadísticamente significativo sobre el porcentaje de infección micorrízica $(\mathrm{P}<0,045, \mathrm{~B}=1,724)$ mientras que la abundancia de hojarasca tiene un efecto negativo $(-0,009)$ sobre la infección.

El valor de la $\mathrm{r}^{2}$ es 0,084 lo cual es un valor muy bajo que nos permite ver que no existe correlación alguna entre las variables independientes (IP, abundancia y profundidad de hojarasca) sobre el porcentaje de infección micorrízica.

\section{Discusión}

Las interacciones entre micorrizas y microorganismos del suelo son determinantes para los ciclos de nutrientes en un ecosistema, afectando el balance entre procesos saprofíticos, patogénicos y simbióticos en el medio ambiente edáfico (Guerrero, 1996) y en la hojarasca (Posada, 2001), además del papel que ejercen en la conservación de los suelos (Miller y Jastrow, 1992). En las raíces analizadas se confirmó la presencia de endomicorrizas al encontrar hifas intramatricales, cuya función principal es la toma y transferencia de nutrientes del sustrato hacia la planta (Sánchez, 1999), y algunas vesículas. De los resultados anteriores puede observarse que no existe un efecto de la pinarización sobre el porcentaje de infección micorrízica, lo cual puede deberse a la temporada en que se realizó el estudio siendo esta temporada seca (febrero) al igual que los valores bajos de infección micorrízica obtenidos para cada uno de los sitios que conforman el bosque 
de pino-encino ya que los factores climáticos afectan la colonización de la raíz, lo que concuerdan con los resultados obtenidos por Moreira (2006) al monitorear la colonización en A. angustifolia en Brasil, concluyendo que durante el tiempo fresco y seco, la colonización de las raíces es baja (25\%), lo contrario de la estación caliente, con más colonización de la raíz (40\%).

El efecto estadísticamente significativo encontrado para la infección micorrízica debido a la profundidad de hojarasca, y ligeramente significativo para la abundancia, puede deberse a que la presencia de hojarasca favorece la actividad microbiológica del suelo. Cuando la vegetación muere, los nutrimentos son descompuestos rápidamente y reincorporados casi inmediatamente al sistema, mediante la asimilación de las plantas vivas. Las raíces toman los nutrimentos con la ayuda de una relación única entre las raíces y las micorrizas que se fijan en las raíces de las plantas y se especializan en incrementar la eficiencia de la toma de nutrimentos que hay en el suelo. Las plantas le dan a cambio al hongo azúcares y lo protegen en sus raíces proporcionándole las condiciones propicias para su desarrollo. La hojarasca constituye uno de los estratos de mayor importancia, presenta la mayor actividad de microorganismos debido a su actividad descomponedora, la cual involucra procesos físicos y químicos que reducen la hojarasca a $\mathrm{CO}_{2}, \mathrm{H}_{2} \mathrm{O}$ y nutrientes minerales, entre los que se encuentran nitrógeno, fósforo, potasio, sodio, calcio, magnesio y azufre. La descomposición de la hojarasca constituye la vía de entrada principal de los nutrientes en el suelo y es uno de los puntos clave del reciclado de la materia orgánica y nutrientes (Vitousek et al., 1994). Las micorrizas constituyen un puente entre la planta y el suelo para la transferencia de nutrientes (Guerrero,
1996). La mineralización de la materia orgánica es realizada por parte de la comunidad microbiana del suelo, cuya actividad está muchas veces limitada por la humedad, la disponibilidad de fuentes de energía y de nutrientes por lo tanto la profundidad de la hojarasca tiene efectos sobre el porcentaje de infección ya que genera las condiciones propicias (en cuanto a temperatura y humedad) para el mejor desarrollo de los hongos micorrízicos.

Aun cuando la acidez no sea un parámetro limitante para el funcionamiento de la simbiosis, sí podría marcar una diferencia positiva en un $\mathrm{pH}$ más cercano al adecuado para el desarrollo de las micorrizas $(4,0-6,0)$; sin embargo, en este trabajo el $\mathrm{pH}$ no es una factor determinante en el grado de infección micorrízica ya que en los tres sitios se obtuvieron porcentajes de infección muy similares aun cuando en el sitio de pino el pH es ligeramente más ácido que en los otros dos sitios.

\section{Conclusiones}

Los porcentajes de infección en un bosque de pino-encino ubicado en el Cerro Ecatepec del municipio de San Cristóbal de las Casas Chiapas fueron de $17,7 \%$ en promedio debido a que el trabajo se desarrolló en una temporada fresca y seca (febrero) encontrándose estructuras como hifas y vesículas. No se observó ningún efecto de la pinarización sobre el porcentaje de infección micorrízica, sin embargo la profundidad de hojarasca presentó un efecto estadísticamente significativo sobre la variable de infección, lo cual puede deberse a que la presencia de hojarasca beneficia la actividad microbiológica del suelo y además la humedad y temperatura de la capa de hojarasca favorece el desarrollo de hongos micorrízicos.

\section{Literatura citada}

Álvarez-Solís, J.D.; León Martínez N.S.

1997. Fertilidad del sueloy sistemas simbióticos. En: Parra Vázquez,M. R. y B. M. DíazHernández (eds.).Los Altos de Chiapas: Agricultura y Crisis Rural. Tomo 1. Los Recursos Naturales. ECOSUR. San Cristóbal de Las Casas, Chiapas, México. Pp. 43-64.

Álvarez-Solís,J.D.; Rosset,P.M.; DíazHB.M.H.; Plascencia V:; RiceR.R. 1998. El impacto de la transformación del paisaje sobre la base productiva de los Altos de Chiapas, México (Avances preliminares). En: Memorias del Seminario sobre manejo de suelos tropicales en Chiapas. El Colegio de la Frontera Sur. San Cristóbal de Las Casas, Chiapas, México. pp. 65-82.
Galindo-Jaimes, L., González-Espinosa. M.; QuintanaAscencio,P.; García-Barrios, L.

2002. Tree composition and structure in disturbed stands with varying dominance by Pinus spp. in the highlands of Chiapas, Mexico. Plant Ecology, 162: 259-272.

García-Barrios, L. E.; González-Espinosa, M.

2004. Change in oak to pine dominance in secondary forests may reduce shifting agriculture yields: experimental evidence from Chiapas, Mexico. Agriculture, Ecosystems and Environment, 102: 389-401. 
Godoy, R.; Romero, R.; Carrillo, R.

1994. Estatus micotrófico de la flora vascular en bosques de coníferas nativas del sur de Chile. Revista Chilena de Historia Natural, 67: 209-220.

González Espinosa, M.

2000. Invasión de Pinus spp. y la conservación de la diversidad florística en Los Altos de Chiapas, México. El Colegio de la Frontera Sur. Informe final SNIB-CONABIO Proyecto No. L329. México, D. F. 105 p.

Guerrero, E. 1996. Micorrizas, recurso biológico del suelo. Fondo FEN de Colombia. Bogotá, Colombia. 208 p.

Miller, R. M.; Jastrow, J. D.

1992. The role of mycorrhizal fungi in soil conservation. En: J. G. Bethlenfalvay y R. G. Linderman (eds.). Micorrhizae in sustainable agriculture. Special publication. No. 54. American Society of Agronomy. Madison, Wisconsin, US. Pp. 29-44.

Moreira, M.; Baretta, D.; Tsai, S.; Nogueira, E.

2006. Spore Density and Toot Colonization by Arbuscular Mycorrhizal Fungi in Preserved or Disturbed Araucaria angustifolia (Bert.) O. Ktze. Ecosystems. Sci. Agric. (Piracicaba, Brazil), 63: 380-385.

Morton, J.B.

1990. Evolutionary relationships among arbuscular mycorrhizal fungi in the Endogonaceae. Mycologia, 82: 192-207.
Morton, J.B.; Benny S.L.

1990. Revised classification of arbuscular mycorrizhal fungi (Zygomycetes): a new order, Glomales, two new suborder, Glomineae and Gigasporineae, and two new families, Acaulosporaceae and Gigaporaceae, with an emendation of Glomaceae. Mucotaxon, 37: 471-491.

Phillips, J.; Hayman, D.

1979. Improved Procedures for Clearing Roots and Staining. parasitic and Vesicular-Arbuscular Mycorrhizal Fungi for Rapid Assessment of Infection. Trans. Br. Mycol. Soc., 1970; 55: 158-160.

Posada, R.

2001. Presencia de propágulos de hongos de micorriza arbuscular en muestras de hojarasca alrededor de dos especies arbóreas en un bosque húmedo tropical. Acta Biológica Colombiana, 6 (1): 24-30.

Sánchez de P. M.

1999. Endomicorrizas en agroecosistemas colombianos. Departamento de Ciencias Básicas, Universidad Nacional de Colombia. Palmira, Colombia. 277p.

Vitousek, P.; Turner, D.; Parton, W.; Sanford R.

1994. Litter Decomposition on the Manua Loa Environmental Matrix, Hawaii: Patterns, Mechanisms and Models. Ecology, 75: 418-429. 
